\title{
Two-Integral Models with Almost Circular Orbits
}

\author{
P. BATSLEER and H. DEJONGHE \\ Sterrenkundig Observatorium, Gent, Belgium
}

\begin{abstract}
We present global dynamical models for bulge-disk systems. The phase-space structure is examined by means of two integral models. The models indicate that in the formation merger argument for ellipticals one cannot always assume that the maximum phase-space density occurs in the center of the system.
\end{abstract}

\section{Introduction}

In a disk, far from the center, stars travel on nearly circular orbits, while closer to the center the very nature of the bulge bring them out of the equatorial plane. As a consequence, one can distinguish two regions with different dynamics in a spiral galaxy. An adequate description of the dynamics of those two regions would require a three integral model. Yet, the construction of such a model is not well defined, because the distribution function is not unique. Therefore we will restrict the analysis to the (unique) two integral distribution function. We argue that a two integral approach is useful because a flat disk has a small extent in the $z$-direction and in the plane of the disk stars travel on nearly circular orbits and thus the velocity dispersion in the $\varpi$ and $z$ direction are both small and from a dynamical point of view where velocities of the order of the circular velocities are relevant, almost equal.

\section{Method}

The orbits the stars travel on in a disk can bring them out of the disk up to a certain height. For a given angular momentum $L_{z}$, any orbit can be populated with binding energy less than the binding energy of the circular orbit with angular momentum $L_{z}$ and corresponding radius $r$. However, we look for bulge-disk systems with a finite extent in the $z$-direction, say $z_{0}$. Hence, for any $L_{z}$ one must introduce a lower limit $S$ for the binding energy below which no motion is allowed. $S$ is the binding energy of the orbit with angular momentum $L_{z}$ that just can reach the height $z_{0}$. Obviously, the farther from the center, the more the stars are confined to the equatorial plane and thus the more the orbits resemble nearly circular orbits, so the less $S$ will differ from the binding energy of the circular orbit at that distance. Closer to the center, the stars must populate those orbits which can produce a bulge structure in configuration space. Hence, orbits different from circular orbits are populated. The energy $S\left(L_{z}\right)$ is given by $S\left(L_{z}\right)=\psi\left(r, z_{0}\right)-L_{z}^{2} /\left(2 r^{2}\left(L_{z}\right)\right)$. The two integral distribution function we propose has the following form:

$$
F\left(E, L_{z}\right)=\sum_{\alpha_{1}, \alpha_{2}, \beta, \gamma} c_{\alpha_{1}, \alpha_{2}, \beta, \gamma} S^{\alpha_{1}}\left(L_{z}\right) e^{-\frac{\alpha_{2}}{S\left(L_{z}\right)}}\left[2 S^{\prime}\left(L_{z}\right) L_{z}^{2}\right]^{\beta}\left[E-S^{\prime}\left(L_{z}\right)\right]^{\gamma}
$$

We introduce the exponential factor to obtain an exponential behaviour for the spatial density, as is observed in the light curves of most disks. To construct the distribution function (1) for a given bulge-disk system, we first select a large number 
of those components which reveal roughly the features of a bulge-disk system. Those components are then fed to a Quadratic Programming routine (see Dejonghe 1989, hereafter QP), which will select in an iterative way a subset of components whose combined density will fit the Van der Kruit-density for that bulge-disk system. The latter density is given by

$$
\rho(R, z)=\exp \left(-\frac{R}{R_{0}}\right) \operatorname{sech}^{2}\left(\frac{z}{z_{0}}\right) .
$$

Obviously, to obtain a physical model, one must add the constraint $F \geq 0$ to the QP-program.

\section{Results}

First of all we found that axisymmetric disks with a large variety of scale parameters $R_{0}$ and $z_{0}$, can be described by two integral models as given in (1). The density (2) is fitted extremely well. As an example, a model for the Galaxy was constructed (see Fig.1, with $R_{0}=2, z_{0}=0.25$ ). Moreover, the QP-method which determines the best components for the distribution function is suitable for this kind of work. The number of components is always small, at most 20.

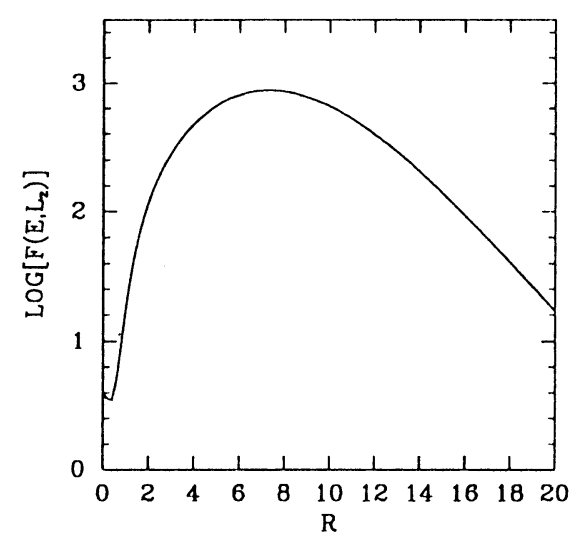

(A)

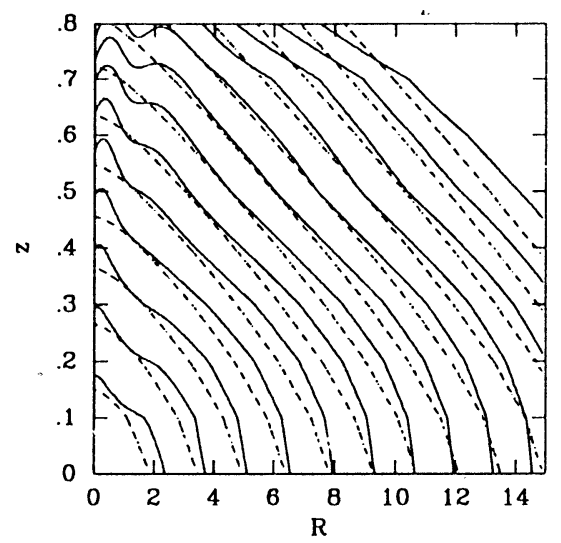

(B)

Fig. 1. (A): Plot of $\log \left[F\left(E, L_{z}\right)\right]$ vs. $R$ along the locus of circular orbit. Notice the maximum phase-space density is not attained in the center and the 3 order of magnitude difference between the distribution function in the center and at its maximum. Notice also the exponential decline! (B): Contourmap of spatial density for the Galaxy model, where the solid line is the two integral density and the dashed line is the van der Kruit density with $R_{0}=2, z_{0}=0.25$

\section{References}

Dejonghe, H., 1989, ApJ, 343, 113.

Gilmore, G., King, I., van der Kruit, P. in The Milky as a Galaxy, Geneva Observatory.

Kruit, P.C. van der, and Searle, L., 1982, AA, 110, 61. 